\title{
A New Approach for Speed Estimation in Induction Motor Drives Based on a Reduced-Order Extended Kalman Filter
}

\author{
Américo Vicente Leite ${ }^{1}$, Member, IEEE, Rui Esteves Araújo ${ }^{2}$, Member, IEEE, and Diamantino Freitas ${ }^{3}$ \\ ${ }^{1}$ School of Technology and Management, Polytechnic Institute of Bragança, Bragança, Portugal, e-mail: avtl@ipb.pt \\ ${ }^{2,3}$ Faculty of Engineering, Porto University, Porto, Portugal, e-mail: raraujo@fe.up.pt and dfreitas@fe.up.pt
}

\begin{abstract}
This paper presents and proposes a new approach to achieve robust speed estimation in induction motor sensorless control. The estimation method is based on a reduced-order Extended Kalman Filter (EKF), instead of a fullorder EKF. The EKF algorithm uses a reduced-order statespace model structure that is discretized in a particular and innovative way proposed in this paper. With this model structure, only the rotor flux components are estimated, besides the rotor speed itself. Important practical aspects and new improvements are introduced that enable us to reduce the execution time of the algorithm without difficulties related to the tuning of covariance matrices, since the number of elements to be adjusted is reduced.
\end{abstract}

Index Terms-Extended Kalman Filter, speed estimation, induction motor, sensorless control.

\section{INTRODUCTION}

In high-performance drives the mechanical speed feedback signal is needed to achieve fast torque response over the entire speed range. Therefore, a speed sensor is required for closed-loop speed or position control in both scalar- and vector-controlled drives. An incremental shaft-mounted optical encoder is usually used for this purpose, but it is an undesirable requirement because the drive cost is increased and additional signal lines are required to connect the encoder and the control electronic system, besides the need for a shaft extension and mounting arrangement.

Due to lower cost and greater reliability without mounting problems, sensorless control methods have been making remarkable developments in the most recent years [1], [2]. Speed estimation methods are being used that avoid the speed measurement set-up and commercial sensorless vector-controlled drives are already available. There are several speed estimation techniques in the literature which are generally classified in [3] as follows: slip calculation; direct synthesis from state equations; model reference adaptive system (MRAS) [4]; speed adaptive flux observer; slot harmonics; injection of auxiliary signal on salient rotor to improve the estimation at low speed [5] and extended Kalman filter (EKF) based methods, [6], [7].

A recent effort in research on sensorless drive control of standard induction motors has been the estimation of rotor speed from the measurement of stator voltages and currents using either the MRAS [4], [8] or the EKF [6], [7] and [9]. This work deals with a new approach for real-time estimation of mechanical speed and rotor flux components by using a reduced-order EKF. Rather than a full-order EKF for speed estimation that also estimates stator current components, besides the estimation of rotor flux components [6], [7], rotor current components [9] or magnetizing current [10], an EKF algorithm based on a reduced-order statespace model is proposed in this paper. In this case only the rotor flux $d q$ components are estimated, besides the rotor speed itself. It is well known that some drawbacks associated to the EKF are the computational effort for real-time applications, the complexity and the hard tuning of the covariance matrices. Important improvements are achieved with respect to all these difficulties using the reduced-order EKF proposed in this paper. In fact, with the $3^{\text {rd }}$-order EKF that is obtained, the dimension of all matrices of the algorithm becomes small enough from a practical point of view. Consequently, on one hand the computational effort is smaller and, on the other hand, the complexity is reduced since the tuning of the algorithm becomes simpler, mainly due to the lower dimension of the state vector. Thus, the genetic algorithm proposed in [6] is not needed any more. Furthermore, if needed, a $2^{\text {nd }}$-order approximation becomes practicable and no more fastidious, with the particular discretization process proposed by the authors in this work.

\section{INDUCTION MOTOR MODEL}

The well-known and established $d q$ dynamic model of a squirrel-cage induction motor is represented by its stator and rotor space phasors voltage equations and stator and rotor flux expressed in terms of stator and rotor currents space phasors [11].

Considering the induction motor equations, in the stator reference frame, and eliminating stator flux and rotor currents space phasors followed by some algebraic manipulations the following sate-space model structure can be obtained, where the notation $\dot{x}$ means $d x / d t$ :

$$
\begin{aligned}
& {\left[\begin{array}{c}
\dot{i}_{s d}^{s} \\
\dot{i}_{s q}^{s} \\
\dot{\psi}_{r d}^{s} \\
\dot{\psi}_{r q}^{s}
\end{array}\right]=\left[\begin{array}{cccc}
a & 0 & b & c \\
0 & a & -c & b \\
d & 0 & -\tau_{r}^{-1} & -\omega \\
0 & d & \omega & -\tau_{r}^{-1}
\end{array}\right]\left[\begin{array}{c}
i_{s d}^{s} \\
i_{s q}^{s} \\
\psi_{r d}^{s} \\
\psi_{r q}^{s}
\end{array}\right]+\left[\begin{array}{cc}
L_{s}^{-1} & 0 \\
0 & L_{s}^{-1} \\
0 & 0 \\
0 & 0
\end{array}\right]\left[\begin{array}{l}
u_{s d}^{s} \\
u_{s q}^{s}
\end{array}\right]} \\
& y=\left[\begin{array}{ll}
i_{s d}^{s} & i_{s q}^{s}
\end{array}\right]^{T}=\left[\begin{array}{llll}
1 & 0 & 0 & 0 \\
0 & 1 & 0 & 0
\end{array}\right]\left[\begin{array}{llll}
i_{s d}^{s} & i_{s q}^{s} & \psi_{r d}^{s} & \psi_{r q}^{s}
\end{array}\right]^{T}
\end{aligned}
$$

with $\quad a=-L_{s}^{-1}\left(R_{s}+L_{M} \tau_{r}^{-1}\right), \quad b=L_{s}^{-1} \tau_{r}^{-1}, \quad c=\omega L_{s}^{\prime-1} \quad$ and $d=L_{M} \tau_{r}^{-1} . \omega$ is the electrical rotor speed in $\mathrm{rad} / \mathrm{s}$ and the 
electrical parameters $\tau_{r}, L_{s}^{\prime}, L_{M}$ and $R_{s}$ represent the rotor time constant, stator transient inductance, mutual inductance and stator resistance, respectively.

This is the type of full-order state-space model used for speed estimation using an EKF like in [6] and [7]. Instead of this full-order model, a reduced-order state-space model structure is derived here for speed estimation. It is obtained rewriting (1a), in the general form $\dot{x}(t)=A x(t)+B u(t)$ and $y(t)=C x(t)$, resulting, respectively, as follows:

$$
\begin{aligned}
& {\left[\begin{array}{l}
\dot{\psi}_{r d}^{s} \\
\dot{\psi}_{r q}^{s}
\end{array}\right]=\left[\begin{array}{cc}
-\tau_{r}^{-1} & -\omega \\
\omega & -\tau_{r}^{-1}
\end{array}\right]\left[\begin{array}{c}
\psi_{r d}^{s} \\
\psi_{r q}^{s}
\end{array}\right]+\left[\begin{array}{cc}
L_{M} \tau_{r}^{-1} & 0 \\
0 & L_{M} \tau_{r}^{-1}
\end{array}\right]\left[\begin{array}{l}
i_{s d}^{s} \\
i_{s q}^{s}
\end{array}\right],} \\
& {\left[\begin{array}{l}
u_{s d}^{s}-\left(R_{s}+L_{M} \tau_{r}^{-1}\right) i_{s d}^{s}-L_{s}^{\prime} i_{s d}^{s} \\
u_{s q}^{s}-\left(R_{s}+L_{M} \tau_{r}^{-1}\right) i_{s q}^{s}-L_{s}^{\prime} i_{s q}^{s}
\end{array}\right]=\left[\begin{array}{cc}
-\tau_{r}^{-1} & -\omega \\
\omega & -\tau_{r}^{-1}
\end{array}\right]\left[\begin{array}{l}
\psi_{r d}^{s} \\
\psi_{r q}^{s}
\end{array}\right],}
\end{aligned}
$$

(2a) being the new state equation and (2b) the new output equation. The state vector and the input and output vectors are given by, respectively:

$$
\begin{aligned}
& x(t)=\left[\begin{array}{ll}
\psi_{r d}^{s}(t) & \psi_{r q}^{s}(t)
\end{array}\right]^{T}=\left[\begin{array}{ll}
x_{1}(t) & x_{2}(t)
\end{array}\right]^{T}, \\
& u(t)=\left[\begin{array}{ll}
i_{s d}^{s}(t) & i_{s q}^{s}(t)
\end{array}\right]^{T}=\left[\begin{array}{ll}
u_{1}(t) & u_{2}(t)
\end{array}\right]^{T}, \\
& y=\left[\begin{array}{ll}
y_{1} & y_{2}
\end{array}\right]^{T},
\end{aligned}
$$

with the outputs $y_{1}$ and $y_{2}$ given by:

$$
\begin{aligned}
& y_{1}=u_{s d}^{s}-\left(R_{s}+L_{M} \tau_{r}^{-1}\right) i_{s d}^{s}-L_{s}^{\prime} i_{s d}^{s}, \\
& y_{2}=u_{s q}^{s}-\left(R_{s}+L_{M} \tau_{r}^{-1}\right) i_{s q}^{s}-L_{s}^{\prime} i_{s q}^{s} .
\end{aligned}
$$

\section{THE REDUCED-ORDER EXTENDED KALMAN FILTER}

Normally, the continuous-time state-space model (1) is discretized using the approximation given by the linear terms of the Taylor's series development which is acceptable if the sampling time $T_{s}$ is small enough. For the reduced-order model (2), suggested in this work, an innovative discretization process is proposed. Thus, the state equation (2a) is discretized using the following and well known relationships [12]:

$$
A_{d}=I+A T_{s}+\frac{A^{2} T_{s}^{2}}{2 !}+\frac{A^{3} T_{s}^{3}}{3 !}+\cdots, B_{d}=B T_{s}+\frac{A B T_{s}^{2}}{2 !}+\frac{A^{2} B T_{s}^{3}}{3 !}+\cdots
$$

but the discrete output equation corresponding to $(2 \mathrm{~b})$ is obtained directly with the first derivative of the stator current components computed by means of a filter of the type [13]:

$$
\left.\dot{i}_{s d, q}\right|_{t=t_{k}} \approx \frac{1}{T_{s}} \sum_{i=0}^{n-1} C_{i} i_{s d, q}\left(t_{k}-i T_{s}\right) .
$$

The only requirement is that the delay introduced by the filter be similar to the one introduced by the approximation used in (3).

For a sampling frequency of $5 \mathrm{kHz}$ the approximations:

$$
\begin{aligned}
& A_{d} \approx I+A T_{s}, \quad B_{d} \approx B T_{s}, \\
& \left.\dot{i}_{s d, q}\right|_{t=t_{k}} \approx \frac{11 i_{s d, q}(k)-18 i_{s d, q}(k-1)+9 i_{s d, q}(k-2)-2 i_{s d, q}(k-3)}{6 T_{s}},
\end{aligned}
$$

have been used successfully with very good results. The matrices $A_{d}$ and $B_{d}$ are the discrete forms of $A$ and $B$. Moreover, the state vector is extended to the rotor speed as a new state variable to be jointly estimated with the rotor flux components, and the extended state vector is given by:

$$
x_{e}(k)=\left[\begin{array}{lll}
\psi_{r d}^{s}(k) & \psi_{r q}^{s}(k) & \omega(k)
\end{array}\right]^{T}==\left[\begin{array}{lll}
x_{e 1}(k) & x_{e 2}(k) & x_{e 3}(k)
\end{array}\right]^{T} .
$$

Assuming $\omega(k+1)=\omega(k)$, that is to say, the speed does not change during the sampling interval, the following stochastic nonlinear model is obtained:

$$
\begin{aligned}
& x_{e}(k+1)=f\left(x_{e}(k), u(k), r_{s}(k)\right)= \\
& =\left\{\begin{array}{l}
\left(1-\tau_{r}^{-1} T_{s}\right) x_{e 1}(k)-x_{e 3}(k) x_{e 2}(k) T_{s}+L_{M} \tau_{r}^{-1} T_{s} u_{1}(k)+r_{s 1}(k) \\
x_{e 3}(k) x_{e 1}(k) T_{s}+\left(1-\tau_{r}^{-1} T_{s}\right) x_{e 2}(k)+L_{M} \tau_{r}^{-1} T_{s} u_{2}(k)+r_{s 2}(k) \\
x_{e 3}(k)+r_{s 3}(k)
\end{array}\right. \\
& \hat{y}=\left[\begin{array}{ll}
\hat{y}_{1}(k) & \hat{y}_{2}(k)
\end{array}\right]^{T}= \\
& =\left\{\begin{array}{l}
h_{1}\left(x_{e}(k), r_{m}(k)\right) \\
h_{2}\left(x_{e}(k), r_{m}(k)\right)
\end{array}=\left\{\begin{array}{l}
-\tau_{r}^{-1} x_{e 1}(t)-x_{e 3}(t) x_{e 2}(t)+r_{m 1}(k) \\
x_{e 3}(t) x_{e 1}(t)-\tau_{r}^{-1} x_{e 2}(t)+r_{m 2}(k)
\end{array}\right.\right. \\
& y=\left\{\begin{array}{l}
y_{1}(k) \\
y_{2}(k)
\end{array}=\left\{\begin{array}{l}
u_{s d}^{s}(k)-\left(R_{s}+L_{M} \tau_{r}^{-1}\right) i_{s d}^{s}(k)-\left.L_{s}^{\prime} i_{s d}\right|_{t=t_{k}} ^{s}(k)-\left(R_{s}+L_{M} \tau_{r}^{-1}\right) i_{s q}^{s}(k)-\left.L_{s}^{\prime} i_{s q}\right|_{t=t_{k}}
\end{array}\right.\right.
\end{aligned}
$$

The EKF can now be applied to the non-linear model described by $(5 \mathrm{a})$ to $(5 \mathrm{c})$ and the state vector is estimated in order to minimize the prediction error, $\varepsilon(k)=y(k)-\hat{y}(k)$.

If a full-order EKF based on the model (1) was used, the dimension of the state vector, $x_{e}$, would be $5 \times 1$ and the system, distribution and output matrices, $\left(A_{d}, B_{d}\right.$ and $\left.C_{d}\right)$ would have dimensions of $5 \times 5,5 \times 2$ and $2 \times 5$, respectively. Furthermore, the system noise covariance matrix would have dimension of $5 \times 5$, what means that we would have 5 diagonal elements to be tuned. On the other hand, the gradient matrices of the EKF algorithm would be $5 \times 5$ and $2 \times 5$.

If the reduced-order EKF based on (5) is used, the dimension of the state vector is $3 \times 1$ and those matrices have the following dimensions: $3 \times 3,3 \times 2,2 \times 3,3 \times 3,3 \times 3$ and $2 \times 3$, respectively. Consequently, the global computational effort is reduced and we need to tune only 3 elements in the diagonal system noise covariance matrix, instead of 5. Actually, we have to tune just 2 elements since, among the 3 ones, 2 of them are equal.

There is another important advantage of the proposed reduced-order model. The matrices $A, B$ and $C$ do not depend neither on the stator parameters nor on the first derivative of the stator current components as can be seen in 
description (2). These parameters and derivatives only appear in the virtual outputs calculated using (5c). So, we can expect a good robustness with respect to these drawbacks since they can be taken into account by tuning conveniently the measurement noise covariance matrix. This means that the errors due to these factors are converted to a lack of confidence in the measures.

\section{EXPERIMENTAL RESULTS}

The above-proposed reduced-order EKF algorithm has been investigated with simulation tests by means of Simulink and the real time validation has been performed using the dSPACE ACE kit based on the DS1103 controller board. A 3kW, 400V, $1430 \mathrm{rpm}$, squirrel-cage induction motor with an incremental encoder was used, loaded by a programmable powder brake, to achieve the experimental results shown in this section.

This work is centered in the reduced-order EKF identification algorithm which was widely tested in real time operation using the dSPACE kit. For the time being the induction motor has been controlled by an ABB frequency converter of the ACS600's family, with open loop speed control.

The speed estimation is based on the stator voltage and current $d q$ components. In the practical implementation, these signals, expressed in the stator reference frame, are filtered with analogue elliptic low-pass pre-filters of fifth order with a $500 \mathrm{~Hz}$ cutoff frequency. A sampling frequency of $5 \mathrm{kHz}$ was used, and the speed estimation is computed with the same cadency.

The electrical parameters of the induction motor were previously estimated by means of the identification techniques proposed by the same authors in the references [14] and [15]. The estimated values of the parameters are presented in table 1.

Table 1: Electrical parameters used in the reduced-order EKF algorithm

\begin{tabular}{cc}
\hline \hline Parameter & Estimated value \\
\hline$\tau_{r}(\mathrm{~ms})$ & 160 \\
\hline$L_{s}^{\prime}(\mathrm{mH})$ & 10 \\
\hline$L_{M}(\mathrm{mH})$ & 200 \\
\hline$R_{s}(\Omega)$ & 2.4 \\
\hline \hline
\end{tabular}

In the implementation scheme, within a simulink block, the third state, corresponding to the rotor speed, was scaled as follows:

$$
x_{e 3}(k)=K \omega(k) \text {, with } K=0.0032 .
$$

This scaling procedure was performed for numerical and practical reasons. On the one hand, the state variables have very different magnitudes since the rotor flux has amplitude close to one and the nominal electrical speed is about 300 $\mathrm{rad} / \mathrm{s}$.
The result of these different sizes is twofold: numerical problems can arise and the states with reduced size can be estimated with significant errors. On the other hand, the speed scaling as in (6) gives another advantage of practical interest: although with different dynamics, the state variables have approximately the same magnitude and the tuning of the diagonal elements of the system covariance matrix become similar. Moreover, since the two components of the rotor flux have the same magnitude and dynamics, only two elements of the system covariance matrix $(Q)$ must be tuned:

$$
Q=\operatorname{diag}\left(\left[\begin{array}{lll}
Q_{11} & Q_{22} & Q_{33}
\end{array}\right], 0\right)
$$

In (7) diag defines a diagonal matrix with $Q_{i j}=0$ and

$$
Q_{33} \approx Q_{22}=Q_{11}=10^{-6}
$$

Furthermore, the measurement noise covariance matrix has been fixed as follows:

$$
R_{m}=\operatorname{diag}\left(\left[\begin{array}{ll}
R_{11} & R_{22}
\end{array}\right], 0\right), \text { with } R_{22}=R_{11}=1
$$

The same value for $R_{22}$ and $R_{11}(=1)$ is possible because the two outputs in $(5 \mathrm{c})$ are similar in terms of magnitude and dynamics. Considering (9) the only elements that must be tuned are $Q_{22}\left(=Q_{11}\right)$ and $Q_{33}$ which control the dynamics of the reduced-order EKF with respect to flux and speed, respectively. This is very important because the EKF tuning is performed in an ah hoc way that is much simpler with the reduced-order EKF proposed in this paper than with a full-order one.

The state vector and the state covariance matrix were initialized, respectively, as follows:

$$
\begin{aligned}
& x_{e}(0)=\left[\begin{array}{lll}
0 & 0 & 0
\end{array}\right]^{T}, \\
& P(0)=\operatorname{diag}\left(\left[\begin{array}{lll}
10^{-8} & 10^{-8} & 10^{-8}
\end{array}\right], 0\right) .
\end{aligned}
$$

\section{A. Transient versus steady-state performance}

The effect of the state covariance matrix given by (11) is not important but in the first instants. On the contrary, the system covariance matrix $Q$ plays an important role in the behavior of the algorithm mainly the element $Q_{33}$ which controls directly the gain of the EKF relatively to the estimated speed. Fig. 1 shows the effect of $Q_{33}$ in the estimated speed when it is selected around the value $Q_{11}=10^{-6}\left(=Q_{22}\right)$.

The analysis of fig. 1 enables us to conclude that the performance of the reduced-order EKF is a compromise between transient and steady-state conditions of the motor operation. This means that the error in the estimated speed can be very low in transient conditions being higher in steady-state operation and vise-versa.

The results of fig. 2 show the performance of the reduced-order EKF in continuous dynamic operation. The speed reference consists of a square waveform with fre- 
quency about $1.7 \mathrm{~Hz}$. The following system covariance matrix was used:

$$
Q=\operatorname{diag}\left(\left[\begin{array}{lll}
10^{-6} & 10^{-6} & 10^{-6}
\end{array}\right], 0\right) .
$$

In fig. 3 is shown the speed reference (square wave with period of $4 \mathrm{~s}$ and $30 \mathrm{rpm}$ of amplitude), and measured and estimated speeds. The load torque is about $10 \mathrm{Nm}$.
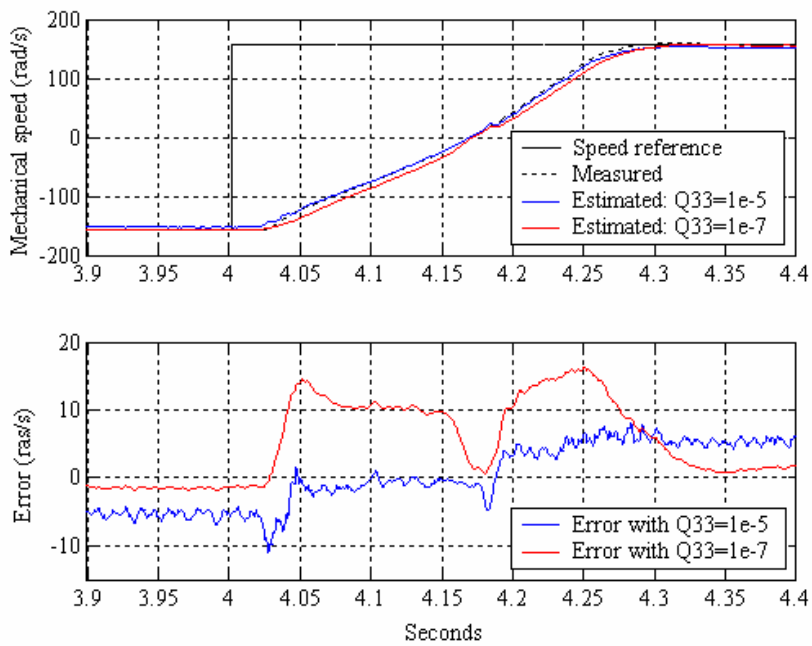

Fig. 1 Transient versus steady-state performance of the reduced-order EKF. Above: reference, measured and estimated speeds in a speed reversal from -1500 to $+1500 \mathrm{rpm}$. Bellow: Speed error in $\mathrm{rad} / \mathrm{s}$.

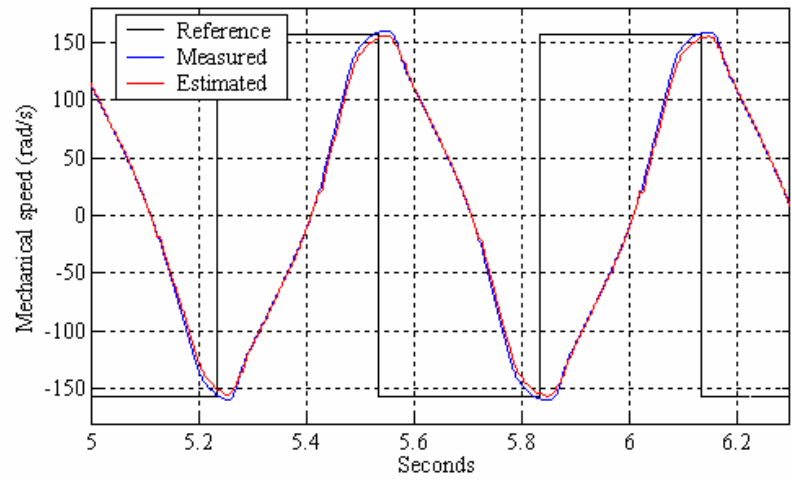

Fig. 2 Performance of the reduced-order EKF with speed reversals between $\pm 1500 \mathrm{rpm}$. The frequency of the speed reference is $1.7 \mathrm{~Hz}$.

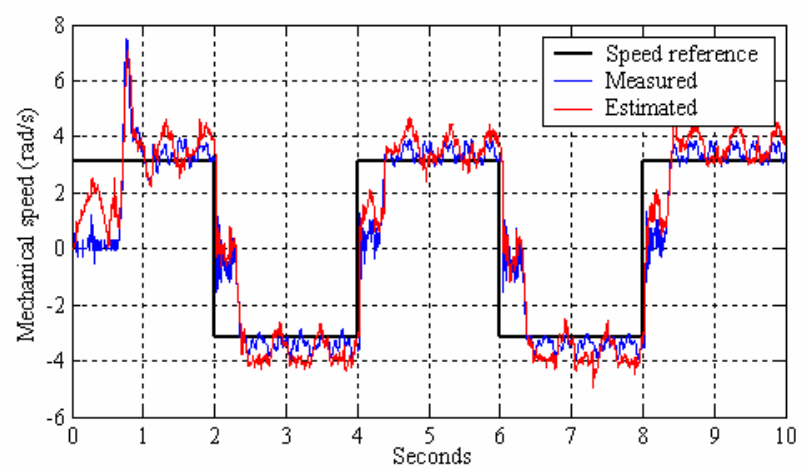

Fig 3 Performance of the algorithm with speed reversals between $\pm 30 \mathrm{rpm}$. The period of speed reference is $4 \mathrm{~s}$ and the load torque is $10 \mathrm{Nm}$.

\section{B. Sensitivity to parameter variation}

A series of tests were achieved in order to evaluate the behavior of the algorithm with respect to parameter variation. The performance depends on speed range and load torque. At high speeds, above some hundreds of rpm, the speed error is reduced (less than 3.5\%) even with full load (15 Nm) as shown in fig. 4(a) to 4(d). The electrical parameters were varied in a range of $\pm 50 \%$ around their estimated values, with a constant speed of $1500 \mathrm{rpm}$, with nominal torque.

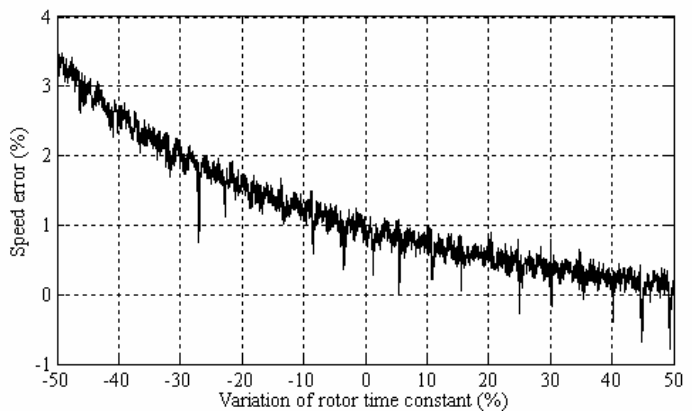

(a)

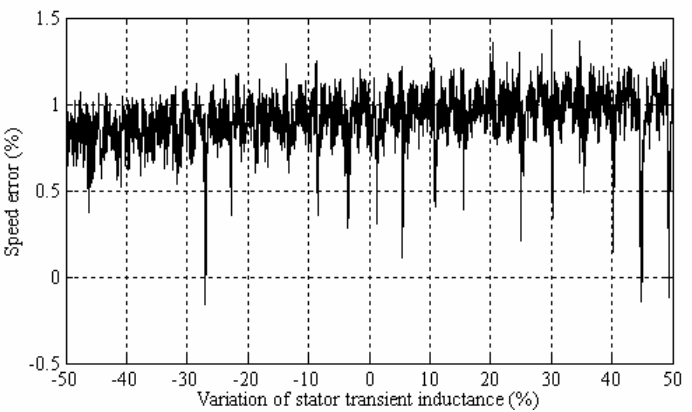

(b)

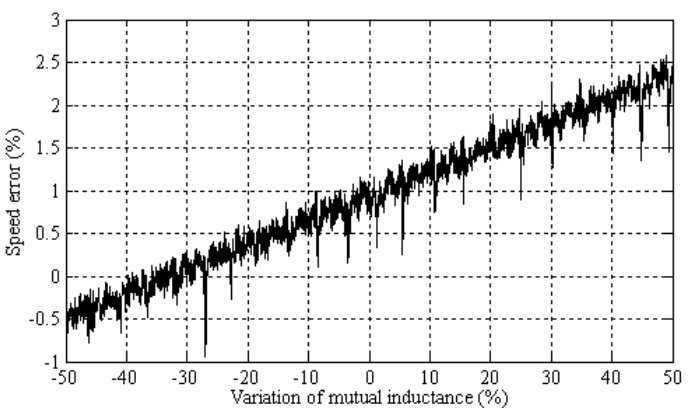

(c)

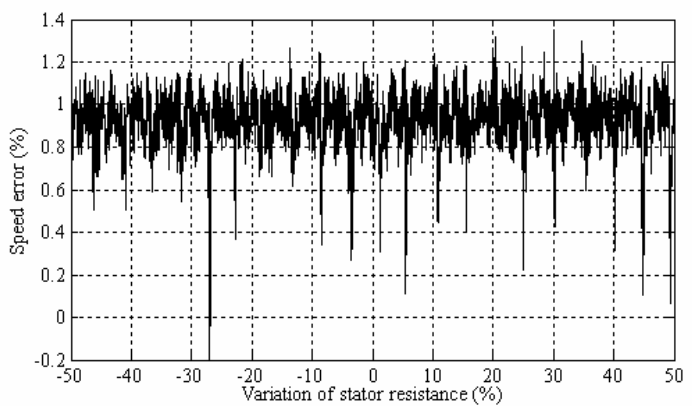

(d)

Fig. 4 Performance of the reduced-order EKF at $1500 \mathrm{rpm}$, under full load, when the electrical parameters are varied from $-50 \%$ to $+50 \%$. Error $(\%)$ in the estimated speed for variation of: (a) rotor time constant, (b) stator transient inductance, (c) mutual inductance and (d) stator resistance. 
The higher errors happen under full load and at low speeds as shown in fig. 5(a) to 5(d). In this case the electrical parameters were varied in a range of $\pm 50 \%$ but with a constant speed of $100 \mathrm{rpm}$.

\section{Performance in several conditions of operation}

Fig. 6 to 9 below, show the performance of the EKF algorithm in a series of transients and steady-state zones, including load and no load, as well as zero speed.

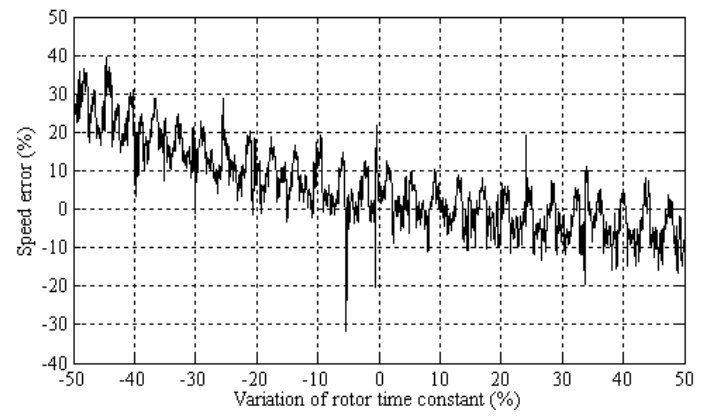

(a)

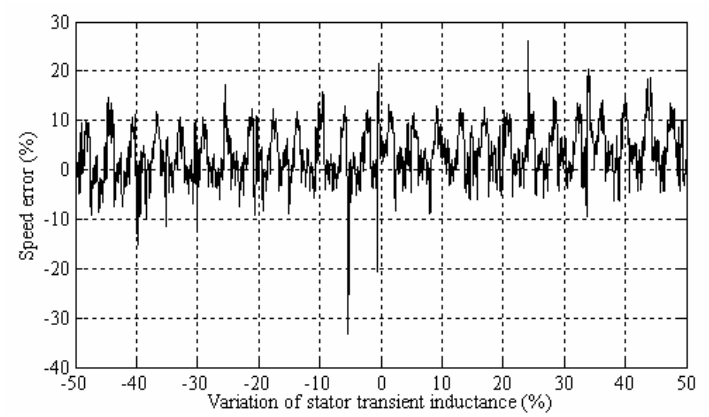

(b)

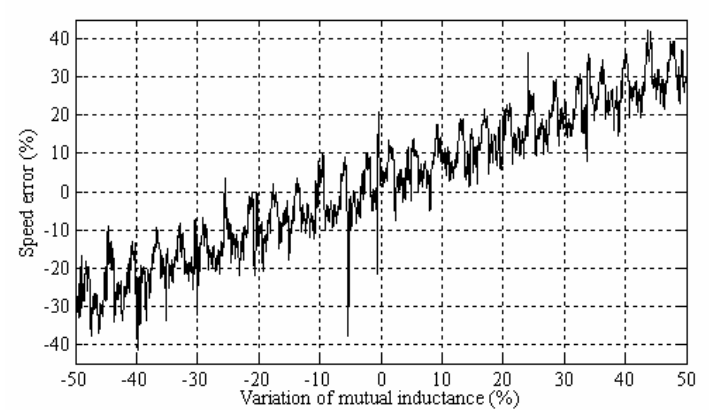

(c)

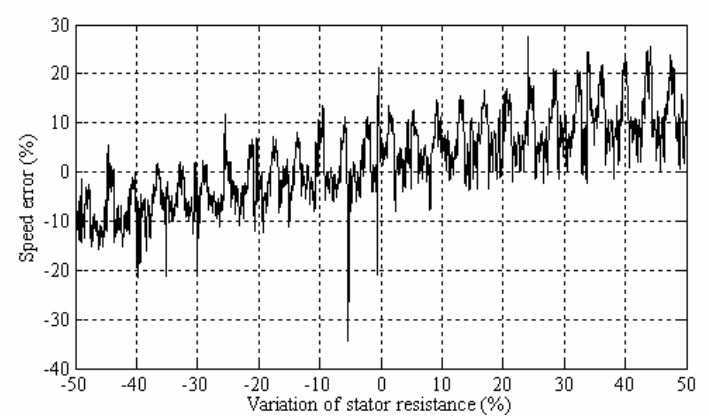

(d)

Fig. 5 The same as in fig. 4 but with the motor running at a constant speed of $100 \mathrm{rpm}$.

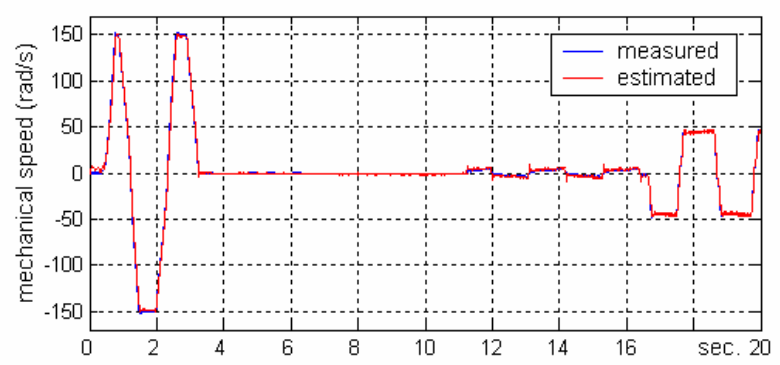

(a)

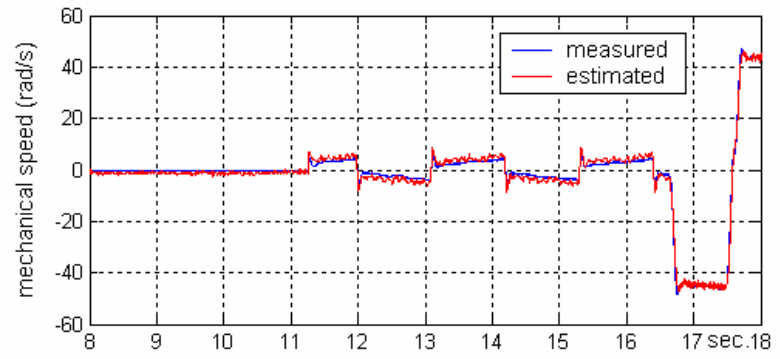

(b)

Fig. 6 (a) Test with inversions between nominal speed, followed by a zero speed command and new inversions between $\pm 100 \mathrm{rpm}$ and $\pm 500 \mathrm{rpm}$. (b) Zoom in. Load torque of $10 \mathrm{Nm}$.

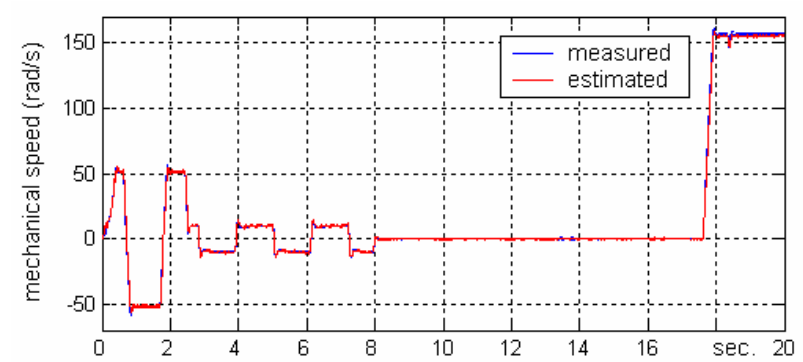

(a)

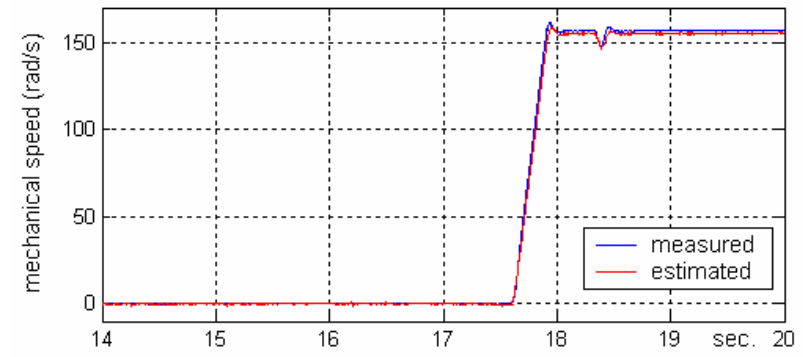

(b)

Fig. 7 (a) Test with inversions between $\pm 500 \mathrm{rpm}$ and $\pm 100 \mathrm{rpm}$, followed by a zero speed command and acceleration to nominal speed. (b) Zoom in. No load torque.

One important aspect that was also investigated is the range of parameter variation that keeps the convergence of the algorithm. All parameters, each one in his turn, were varied during real time operation under the worst conditions, that is to say, with continuous reversals between $\pm 100 \mathrm{rpm}$ with nominal torque. The results are summarized in table 2.

The speed error becomes very high with a rotor time constant as low as some tens of ms and the same occurs for both limits of the mutual inductance. The estimated speed becomes very noisy for values of stator transient inductance above $40-50 \mathrm{mH}$. The algorithm becomes quickly unstable when the stator resistance is higher than $3.4 \Omega$. 


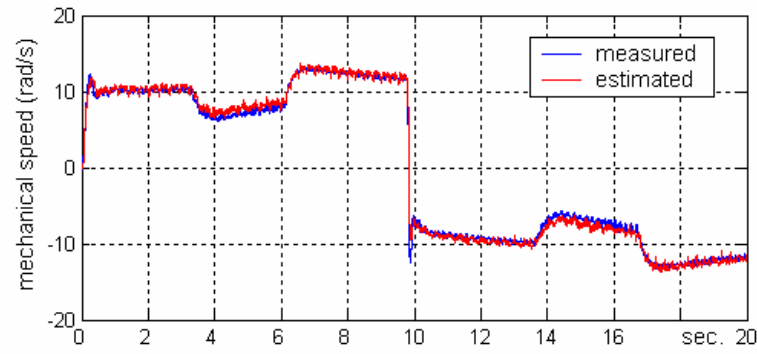

(a)

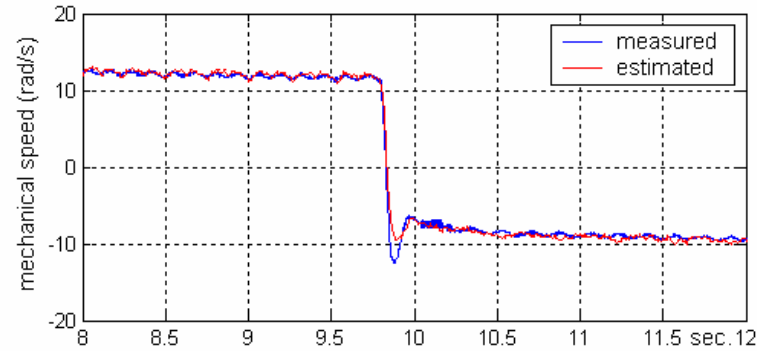

(b)

Fig. 8 (a) Test with a start-up followed by an inversion between $\pm 100 \mathrm{rpm}$, with no load torque. (b) Zoom in. Perturbations with a load torque of $10 \mathrm{Nm}$ were included in the steady-state zones.

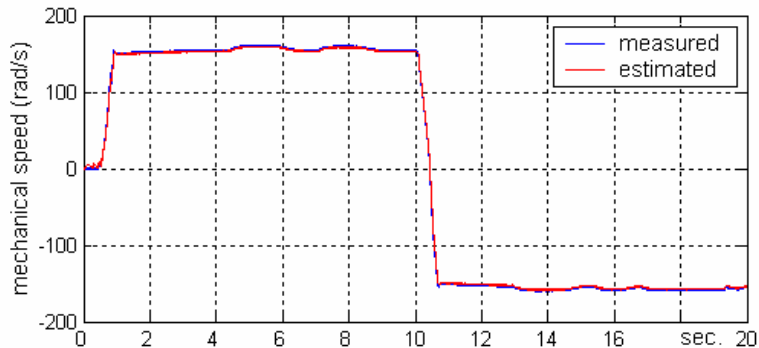

(a)

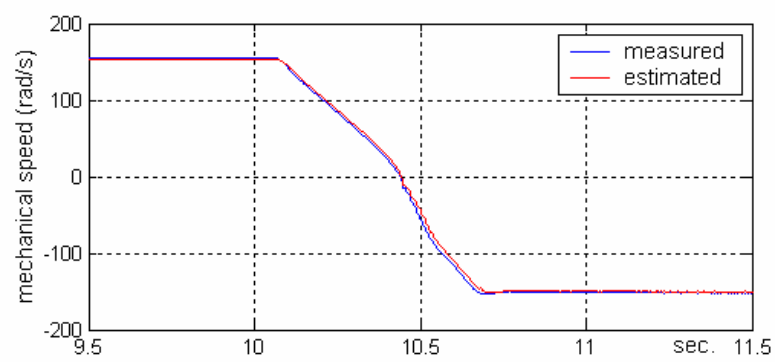

(b)

Fig. 9 (a) Test with a start-up followed by an inversion between \pm 100 rpm, with load torque of $10 \mathrm{Nm}$. (b) Zoom in. Perturbations with no load torque were included in the steady-state zones.

Table 2: Parameter variation that keeps the convergence of the algorithm with continuous reversals between $\pm 100 \mathrm{rpm}$ with nominal torque.

\begin{tabular}{cc}
\hline \hline Parameter & Range of variation \\
\hline \hline$\tau_{r}(\mathrm{~ms})$ & $40 \rightarrow 500$ \\
\hline$L_{s}^{\prime}(\mathrm{mH})$ & $0 \rightarrow 50$ \\
\hline$L_{M}(\mathrm{mH})$ & $0 \rightarrow 350$ \\
\hline$R_{s}(\Omega)$ & $0 \rightarrow 3.4$ \\
\hline \hline
\end{tabular}

\section{CONCLUSIONS}

A new approach to achieve high-performance and robust speed estimation in induction motor drives, namely, in sensorless control, utilizing a reduced-order EKF algorithm, has been presented in this paper.

A series of simulation and experimental tests have demonstrated a good performance with respect to the parameter's variation and noise in the full range of load torque and speed. However, at very low speeds (below a few hundreds of rpm) the error in the estimated speed can become significant if the parameters are not precisely known mainly the mutual inductance. A series of real time tests using the dSPACE ACE kit based on DS1103 controller board proved the conclusions of the simulation research and some experimental results were presented.

The computational effort is reduced and the tuning of the algorithm is not hard any more when compared with the full-order EKF and no more complex and time-consuming procedures are required for this purpose as happened in [6].

\section{REFERENCES}

[1] K. Rajashekara, A. Kawamura, K. Maytsuse, Sensorless Control of AC Motor Drives - Speed and Position Sensorless Operation, IEEE Press, 1996.

[2] P. Vas, Sensorless Vector and Direct Torque Control, Oxford University Press, New York, 1998.

[3] B. K. Bose, Modern Power Electronics and AC Drives, Prentice Hall PTR, Upper Saddle River: 2002.

[4] M. Rashed, F. Stronach, P. Vas, "A Stable MRAS-Based Sensorless Control Induction Motor Drive at Low Speeds", in Proc. of the IEEE-IEMDC Conference, 2003, in CD-ROM.

[5] D. Drevenšek,D. Žarko, T. Lipo, "A study of Sensorless Control of Induction Motor at Zero Speed Utilizing High Frequency Voltage Injection", EPE Journal, vol. 13, n. ${ }^{\circ}$ 3, Aug. 2003, pp. 7-11.

[6] K. L. Shi, T. F. Shan, Y. K. Wong, S. L. Ho, "Speed Estimation of an Induction Motor Drive Using an Optimized Extended Kalman Filter”, IEEE Trans. Ind. Electron., vol. 49, n. ${ }^{\circ}$ 1, Feb. 2002, pp. 124133.

[7] Y. R. Kim, S. K.. Sul, M. H. Park, "Speed Sensorless Vector Control of Induction Motor Using Extended Kalman Filter", in Sensorless Control of AC motor Drives, IEEE Press, New York: 1996, pp. 215 223.

[8] E. Levi, M. Wang, "A Speed Estimator for High Performance Sensorless Control of Induction Machines Above Base Speed", in Proc. of the EPE-PEMC Conference, 2002, in CD-ROM.

[9] J. K. Al-Tayie, P.P. Acarnley, "Estimation of Speed, Stator Temperature and Rotor Temperature in Cage Induction Motor Drive Using the Extended Kalman Filter Algorithm", IEE Proc.-Electr. Pawer Appl., vol. 144, n. ${ }^{\circ}$ 5, Sep., 1997, pp. 301-309.

[10] Sensorless Control with Kalman Filter on TMS320 Fixed-Point DSP, Texas Instruments Europe, Literature n. ${ }^{\circ}$ BPRA057, July, 1997.

[11] P. Vas, Parameter Estimation, Condition Monitoring, and Diagnosis of Electrical Machines, Oxford University Press, New York: 1993.

[12] F. L. Lewis, Applied Optimal Control Estimation - Digital Design and Implementation, Prentice Hall, New York: 1992.

[13] A. J. L. Harrison, D. P. Stoten, "Generalized Finite Difference Methods for Optimal Estimation of Derivatives in real-Time Control Problems", in Proc. Instn Mech Engrs, vol. 209, 1995, pp 67-78.

[14] A. V. T. Leite, R. M. E. Araújo, D. R. S. Freitas, "A New Online Identification Methodology for Flux and Parameters Estimation of Vector Controlled Induction Motors", in Proc. of the IEEE-IEMDC 2003 Conference, 2003, in CD-ROM.

[15] A. V. T. Leite, R. M. E. Araújo, D. R. S. Freitas, “A Boot-Strap Estimator for Joint Flux and Parameters Online Identification for Vector Controlled Induction Motor Drives", in Proc. of the EPE 2003 Conference, 2003, in CD-ROM. 